\title{
Assessment of the Protective Capacity of Nanosomes of Quercetin in an Experimental Model of Parkinson's Disease in the Rat
}

\section{Marcela Díaz, Lucía Vaamonde and Federico Dajas*}

Department of Neurochemistry, Instituto de Investigaciones Biológicas Clemente Estable, Montevideo, Uruguay

${ }^{*}$ Corresponding author: Federico Dajas, Department of Neurochemistry, Instituto de Investigaciones Biológicas, Clemente Estable, Avda Italia 3318, 11600 Montevideo, Uruguay, Tel: 59824871616 123; E-mail: fdajas@gmail.com

Rec date: September 15, 2015 Acc date: September 24, 2015 Pub date: September 30, 2015

Copyright: @ 2015 Díaz M, et al. This is an open-access article distributed under the terms of the Creative Commons Attribution License, which permits unrestricted use, distribution, and reproduction in any medium, provided the original author and source are credited.

\begin{abstract}
Quercetin, a representative flavonoid, is a potent antioxidant and anti-inflammatory molecule that modulates cellular signals and promotes the expression of survival proteins. In spite of this pharmacological profile, the administration of quercetin to experimental Parkinson's Disease (PD) models has shown controversial results. Low plasma and brain bioavailability following acute administration due to high metabolization rates and poor crossing of the blood brain barrier have been proposed as explanations. In an attempt to surpass these difficulties, we developed lecithin/cholesterol/2-hydroxypropyl- $\beta$-cyclodextrin nanosomes of quercetin (NSQ), to test whether the use of an appropriate vehicle can provide metabolic protection and ease the delivery to the brain to achieve active brain concentrations that assure the survival of neurons in a model of PD. As an in vivo experimental test of the effectiveness of NSQ, we administered it after the 6-hydroxydopamine (6-OHDA) injection in the Substantia Nigra $(\mathrm{SN})$ in a model of PD in rats. The nanosomes were effective in delivering quercetin to the brain via both intravenous and intraperitoneal routes of administration and prevented the increase in malondialdehyde in the SN provoked by the lesion.
\end{abstract}

The decrease in striatal dopamine levels following 6-OHDA administration in SN was significantly ameliorated following NSQ treatment one hour after the toxin injection. The empty nanosomes prevented corpus striatum (CS) dopamine decrease when given in multiple, repeated doses at 1, 24, 48 and 72 hours after 6 OHDA. In conclusion, nanosomes of quercetin achieve brain concentrations of free quercetin and show protective effects in experimental Parkinson's Disease at the terminal field (CS) of lesioned Substantia Nigra neurons that did not recover after treatment.

Keywords: Quercetin; Parkinson's disease; Nanosomes; Dopamine

\section{Introduction}

Parkinson's Disease is the second most important neurodegenerative disorder after Alzheimer's disease [1]. Key pathological features include the degeneration of dopaminergic neurons in the substantia nigra $(\mathrm{SN})$ pars compacta $(\mathrm{pc})$ that is associated with intracytoplasmic inclusions known as Lewy bodies.

Currently, the primary treatment consists of a dopamine (DA) replacement strategy based on levodopa that provides temporary benefits but loses efficacy after 5-10 years at which point some patients develop dyskinesias and behavioural abnormalities [2]. In this context, important research efforts are dedicated to understand the etiology of $\mathrm{PD}$ and to develop therapies that can provide symptomatic alleviation or slow the natural progression of the disease.

Although the pathogenic mechanisms that underlie PD remain unknown, it is widely acknowledged that both mitochondria and oxidative stress are important factors in the pathogeneses of both the sporadic and genetic forms of the disease $[3,4]$.

On the other hand, important evidence for the antioxidant effects of small molecules, such as natural polyphenols, has come to occupy a central role because of their therapeutic potential as neuroprotective or lead molecules. Flavonoids are the largest group of polyphenols and are present in beverages obtained from plants, fruits and vegetables, such as olive oil, red wine and tea [5]. The use of flavonoid-rich plant or food extracts in human and animal dietary supplementation studies have resulted in improvements in cognitive function at the experimental and clinical levels [6-9]. Although epidemiological studies have shown the advantages of flavonoid-rich food in the prevention of neurodegeneration, demonstration of the therapeutic effects of independent flavonoids in PD has been controversial. While flavonoids, such as tangeretin [10] and naringenin [11], have shown to be neuroprotective in experimental $\mathrm{PD}$, representative flavonoids, such as quercetin, have shown little or no protective effects in in vivo models $[12,13]$. Low plasma and brain bioavailability following acute administration, high metabolization rates and poor crossing of the blood brain barrier have been advanced as explanations for these results [9]. The poor brain bioavailability of quercetin is a pharmacological challenge that requires the use of drug delivery systems to improve its access to the brain and other organs in acute treatments $[14,15]$.

Since their discovery in the 1960's, a great variety of liposomes have been utilized for drug delivery of multiple compounds [16] and liposomes are currently the nano-carrier system most widely studied for use as a vehicle for flavonoids [17]. Encapsulation would mask the water insoluble nature of quercetin, protecting it from metabolism, prolonging its circulation and increasing the half-life. After repeated tests, we developed lecithin/cholesterol/2-hydroxypropyl- $\beta$ cyclodextrin (2HBCD) nanosomes that assured a good bioavailability of quercetin. Preliminary studies have shown very good and safe 
bioavailability, improving brain function in severely hypoxic new-born piglets after intravenous administration $[18,19]$. The aim of the present study was to test the effectiveness of a preparation of quercetin nanosomes to protect of the brain. To this objective, we have utilized an experimental model of Parkinson's Disease. The knowledge gained with this approach would be of value for the putative therapeutic use of flavonoids or flavonoid derivatives in neurodegenerative diseases.

\section{Material and Methods}

\section{Chemicals}

Urethane, 6-OHDA, quercetin, luteolin, DA, thiobarbituric acid, 1,1,3,3-tetramethoxypropane (TBA), butylated hydroxytoluene (BHT), sodium dodecyl sulfate, and sodium octyl sulfate were obtained from Sigma Chemical Co. (St. Louis, MO, USA). The antibodies used in the Immunohistochemical assays were obtained from Millipore (polyclonal primary anti-TH) and Thermo Scientific (secondary DyLight 488). All other reagents used in this study were obtained from standard suppliers and were of analytical grade or better.

\section{Nanosomal formulation}

Lecithin/cholesterol/2-hydroxypropyl- $\beta$-cyclodextrin nanosomes of quercetin were obtained in volumes of $20 \mathrm{ml}$ in a reactor device where the mixture reacted under established conditions of flow, pressure and temperature $\left(60^{\circ} \mathrm{C}\right)$ in physiological solution after initial sonication of the lecithin/cholesterol mixture for $24 \mathrm{~h}$ (Patent pending, PCT/ IB2013/059067). Stabilizing tests and electron microscopic and atomic force microscope analyses of nanosomes were performed.

\section{Animals}

The Committee of Bioethics of the Clemente Estable Biological Research Institute (IIBCE) approved all of the procedures involving the use of animals described in this study. Male Sprague-Dawley rats weighing 270-300 g were bred in the vivarium of the IIBCE, were provided ad libitum access to food and water and were housed in groups of six in a temperature controlled environment on a 12-h light/ dark cycle.

The rats were randomly divided into various experimental groups, and there was no significant difference in the average body weights of the groups.

\section{Bioavailability of quercetin}

Seven experimental groups ( $\mathrm{n}=4$ per group) were studied. One of these groups was used as a control to determine whether there were endogenous levels of quercetin in the brain. The other groups were treated with i.p. or i.v. NSQ and evaluated 1, 2 or $3 \mathrm{hrs}$ after the administrations.

\section{Administration of the nanosomal preparation of quercetin}

Intravenous injection: After the animals were anesthetized via i.p. injections of urethane, the femoral vein in the hind paw was exposed, and a catheter (G26 × $19 \mathrm{~mm}, \mathrm{BD}$ Neoflon) connected to a microinjection pump (CMA/100 microdialysis) was introduced into the vein under a magnifying glass. A constant flow of $55 \mu \mathrm{l} / \mathrm{min}$ was used for the administration of the appropriate dose of the preparation $(10 \mathrm{mg} / \mathrm{kg})$. The time points selected for the determinations of the concentrations of quercetin in the brain were taken relative to the completion of the administrations.

Intraperitoneal injection: The same preparation at the same dose was administered via i.p. injection to awake animals. According to the different protocols (Figure 1), trans-cardiac perfusions with saline were performed under anaesthesia (urethane) at 1,2 or $3 \mathrm{hrs}$ after the end of the administrations, and the brains were removed. The tissues were stored at $-80^{\circ} \mathrm{C}$ until analysis.

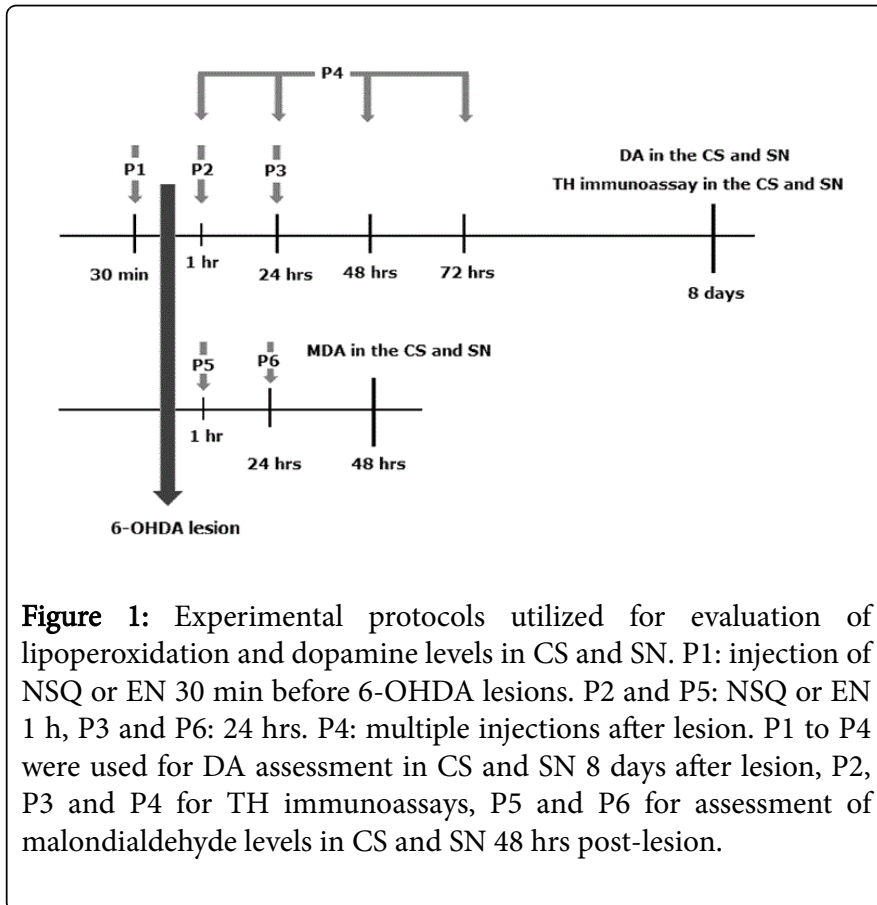

\section{Analysis of quercetin}

Quercetin was assessed by high performance liquid chromatography with electrochemical detection (HPLC-ED). The brains, which had been maintained at $-80^{\circ} \mathrm{C}$, were brought to room temperature, and 400 $\mathrm{mg}$ of midbrain including the CS were separated. The cortex was excluded, and $1400 \mu \mathrm{l}$ of a mixture of methanol, BHT (0.25 M), EDTA $(0.1 \mathrm{M}), 200 \mu \mathrm{l}$ of $0.1 \mathrm{M}$ perchloric acid and $100 \mu \mathrm{l}$ luteolin was added (luteolin was used as the internal standard for subsequent calculation of the percent recovery of quercetin). The samples were homogenized (10 pulses) on ice and stirred for $10 \mathrm{~min}$. The samples were then centrifuged for $15 \mathrm{~min}$ at $5000 \mathrm{~g}$, and $1 \mathrm{ml}$ of supernatant diluted in $1600 \mu \mathrm{l}$ of bidistilled water was taken. The samples were filtered through SPE columns (Phenomenex, Strata X-cw33 $\mu, 60 \mathrm{mg} / 3 \mathrm{ml}$ polymeric weak cation) through which quercetin was extracted in $2 \mathrm{ml}$ methanol. The methanol was evaporated from the samples by nitrogen gas. Quercetin and luteolin were re-suspended, concentrated in methanol/phosphate buffer (0.5\%), mixed and centrifuged for $15 \mathrm{~min}$ at $15000 \mathrm{rpm}$. Fifty microliters of the supernatant was injected into the HPLC-ED.

The HPLC-ED consisted of an infusion pump (Bioanalytical Systems, PM-80), an electrochemical detection system LCEC Epsilon and a solid phase column (Phenomenex C-18 $150 \times 4.6 \mathrm{~mm}, 5$ micron). A mobile phase composed of $50 \%$ phosphate buffer $(0.5 \%)$ and $50 \%$ methanol at $\mathrm{pH} 2$ was used. The running conditions were as follows: flow rate $1 \mathrm{ml} / \mathrm{min}, 100 \mathrm{nA}$ sensitivity, and oxidation potential of $+600 \mathrm{mv}$. 
The peaks of the corresponding concentrations of the different components of the samples were quantified with CromGraph software (BAS) version 2.34 .00 (2001). Standards of known concentrations of quercetin and luteolin $(75 \mathrm{ng} / \mathrm{ml}$ dissolved in DMSO and $750 \mathrm{ng} / \mathrm{ml}$ dissolved in methanol, respectively) were used to determine the percentages of recovered quercetin and the quercetin concentrations in brain as a function of time.

\section{Intranigral injection of 6-OHDA}

Experimental procedures were performed according to our previous study (19). Animals were anaesthetized with halothane (Fluothane, Zeneca) and placed in a D. Kopf stereotaxic frame. Through a skull hole, the needle $(0.022 \mathrm{~mm}$ o.d., $0.013 \mathrm{~mm}$ i.d.) of a Hamilton syringe $(5 \mu \mathrm{l})$, attached to a micro-injection unit (D. Kopf), was lowered to the $\mathrm{SN}$. Coordinates $(\mathrm{H},-4.8 ; \mathrm{L},-2.2 ; \mathrm{V},-7.2)$ were determined from bregma, according to the atlas of Paxinos and Watson [20].

A total of $2.0 \mu \mathrm{l}$ of a 6 -OHDA solution ( 3 and $5 \mu \mathrm{g} / \mu \mathrm{l}$ for the 6 - and 10- $\mu$ g doses) was injected for $4 \mathrm{~min}$ and the needle was slowly withdrawn, allowing the drug to diffuse for another minute. Body temperature was maintained at $37^{\circ} \mathrm{C}$ using a temperature control system.

\section{Lipoperoxidation levels}

Six experimental groups ( $\mathrm{n}=4$ per group) were used. The first, control group, did not receive 6 OHDA or treatment and was used to determine the basal levels of MDA. The second group was only 6 OHDA, and groups 3 to 6 were 6 OHDA injected and treated with NSQ or EN 1 or 24 hrs after (P5 and P6) (Figure 1).

The groups of animals used for these experiments were injected unilaterally with 6-OHDA as described above. After dissection of the $\mathrm{SN}$ and CS in cold conditions, the samples were processed as follows:

The CS of each hemisphere was separately placed in $300 \mu$ of acetic acid to minimize the spontaneous lipoperoxide formation.

For the SN, three samples per tube were immersed in $300 \mu \mathrm{l}$ of acetic acid (separately left and right hemispheres). All tissues were stored at $-80^{\circ} \mathrm{C}$. Previously to assessment, frozen tissue was thwarted at room temperature. Afterwards, the tissue was homogenized in ice using a Teflon homogenizer tip (10 pulses), and $50 \mu$ l were separated for protein assessment via the bicinchoninic acid assay (BCA).

The remaining homogenate was centrifuged at $800 \mathrm{~g}$ for 15 minutes at $4^{\circ} \mathrm{C}$. Next, $50 \mu \mathrm{l}$ of the supernatant was mixed with $350 \mu \mathrm{l}$ of acetic acid and $600 \mu \mathrm{l}$ of $0.8 \%$ TBA (dissolved in $20 \%$ acetic acid pH 3.5, concentration: $8 \mathrm{mg} / \mathrm{ml}$ ). For the calibration curve, $400 \mu \mathrm{l}$ of each dilution were mixed with $600 \mu$ of TBA.

All tubes were incubated for 30 minutes at $95^{\circ} \mathrm{C}$. The samples were then cooled for 6 minutes on ice, and $50 \mu$ of SDS (10\%) was added to remove any lipids that may have interfered with trace quantifications. Finally, the samples were centrifuged at $5000 \mathrm{rpm}$ for 5 minutes at $4^{\circ} \mathrm{C}$, and $200 \mu \mathrm{l}$ of the supernatant were taken for spectrofluorimetric assessment.

\section{Malondialdehyde assessment}

The formation of the adduct TBA-MDA was quantitated in a Varioskan Flash reader (Thermo Scientific) with fluorimetric detection (Ex: $520 \mathrm{~nm}$, Em: $553 \mathrm{~nm}$ ). Acetic acid was used as blank, and its value was subtracted from each measurement to obtain the actual concentration of MDA. The concentration values obtained were corrected by the amount of protein present in the samples as determined by the BCA method.

\section{Dopamine levels}

Nine experimental groups ( $\mathrm{n}=8$ per group) were used. The control group was injected with 6-OHDA, and 8 groups of animals were injected and treated with NSQ or EN according to the 4 protocols of administration (Figure 1).

In all cases, the animals were sacrificed 8 days post-lesion, and the brains were removed quickly on ice. The $\mathrm{SN}$ and CS were dissected and maintained at $-80^{\circ} \mathrm{C}$ until the assessment.

The samples were processed as previously described [19].

\section{Assessment of dopamine}

The quantification of DA and its metabolites in the CS and SN was performed by HPLC-ED, and the values expressed as DA percentages (ng of DA/mg of tissue) in the lesioned side relative to the control side (i.e., lesion/non-lesion side).

For neurochemical analysis rats were decapitated 8 days after 6OHDA injection, brains rapidly removed and the left and right $\mathrm{SN}$ and $\mathrm{CS}$ dissected and kept at $-70^{\circ} \mathrm{C}$. Next day tissue samples were weighed, sonicated in perchloric acid $0.1 \mathrm{M}(200$ and $1000 \mu \mathrm{l}$ for the SN and CS, respectively) and centrifuged $(15,000 \times \mathrm{g})$ for $15 \mathrm{~min}$ at $4^{\circ} \mathrm{C}$.

Then, samples were injected into an HPLC system (PM-80 BAS, USA) equipped with a C-18 column $(5-\mu \mathrm{m}$ particles, $220 \mathrm{~mm} \times 4.6$ $\mathrm{mm}$; BAS, USA) and a electrochemical detector (LC-4C BAS) with oxidation potential at $+0.75 \mathrm{~V}$ (glassy working carbon electrode versus an $\mathrm{Ag} / \mathrm{AgCl}$ reference electrode). The mobile phase was composed of citric acid $(0.15 \mathrm{M})$, sodium octyl sulphate $(0.6 \mathrm{mM}), 4 \%$ acetonitrile and $1.6 \%$ tetrahydrofuran at $\mathrm{pH} 3.0$; with a flow rate of $1.0 \mathrm{ml} / \mathrm{min}$.

\section{Tyrosine hydroxylase immunohistochemistry}

Eight experimental groups ( $\mathrm{n}=8$ per group) were used and consisted of an absolute control, a group of animals that were lesioned with 6OHDA, and 6 groups of lesioned animals that were treated with NSQ or EN according to the three protocols (Figure 1).

Eight days after the lesions, gravity-based transcardial perfusions with heparinized $(0.1 \%)$ saline $(0.9 \%)$ were performed, and the brain tissues were fixed with 4\% PFA solution (dissolved in PBS, pH 7.4) and $5 \%$ sucrose. After post-fixation by immersion in 4\% PFA ( 2 hours) and cryo-protection (sucrose $15 \% 24$ hours $30 \% 48$ hours at $4^{\circ} \mathrm{C}$ ), the brains were stored at $-80^{\circ} \mathrm{C}$ until they were cut at $-20^{\circ} \mathrm{C}$ using a cryostat (Slee Mainz, MEV).

Coronal sections at a thickness of $20 \mu \mathrm{m}$ were prepared at the levels of the SN and CS based on stereotaxic coordinates (20) and collected on gelatinized slides. For both the $\mathrm{SN}$ and $\mathrm{CS}$, serial cuts were performed over a region of $600 \mu \mathrm{m}$ using anteroposterior (AP) coordinates of -5.0 and $+1.2 \mathrm{~mm}$ from bregma, respectively, as the starting points.

Immunohistochemistry techniques were applied to the brain sections as previously described in a report from our laboratory [20-23]. The omission of the primary antibody resulted in a complete loss of immunostaining. 
To detect the presence of $\mathrm{TH}$ in the sections containing the $\mathrm{SN}$ and the CS, a fluorescence microscope (BX 61, Olympus) attached to an Olympus FV300 confocal module ( $\mathrm{Kr}$-Ar laser blue $(488 \mathrm{~nm})$ filter barrier was used.

The Fluoview (Olympus, version 4.3) software was used for the acquisition and analysis of the confocal images of which 6 slices were acquired at a $2 \mu \mathrm{m} \mathrm{z}$ step for each section. All images assessed in this study were acquired at a resolution of $1024 \times 1024$ pixels using a $40 \mathrm{x}$ objective lens (NA=0.75 UPlanFLN, Olympus).

In the $\mathrm{SN}$, the quantifications of the TH-positive neurons were performed based on the maximum-intensity projection images. In the $\mathrm{CS}$, the integrated optical densities (IODs), which are equivalent to the optical densities (i.e., fluorescence intensities) per area $\left(\mu \mathrm{m}^{2}\right)$ were assessed from simple images of single optical planes.

\section{Quantification of tyrosine hydroxylase-positive neurons in the SN}

For this analysis, 6 slices of three separate AP regions per hemisphere per rat were selected. The neuronal counts were performed manually through the entire lateral axis of the SN using the Image J program with the Cell counter for image processing plugin (1.45 s, Wayne Rasband, National Institutes of Health, USA). The neurons of the 6 sections were summed to obtain the final number of TH-positive neurons in $\mathrm{SN}$ per animal.

\section{Quantification of tyrosine hydroxylase IOD in the CS}

For this study, 4 slices from two separate regions (lateral and medial) in the AP axis per hemisphere per rat were taken. Using the Count/Size tool of the Image Pro Plus software (version 5.1.0.20, Media Cybernetics, USA), the corresponding IODs were quantified for three areas $\left(5776 \mu \mathrm{m}^{2} / \mathrm{each}\right)$ of the images that were selected to prevent striations. These values were then averaged to obtain the IOD for each image.

\section{Data analysis}

The data are expressed as the means + the standard deviations (SDs). Quercetin concentrations are presented as ng/g of tissue, and malondialdehyde, dopamine levels, TH-positive neurons and IODs are presented as the percentages in the lesions relative to the non-lesioned SN or CS; i.e., the non-lesioned side was taken as $100 \%$.

Statistical significance was determined via analyses of variance (ANOVAs) followed by multiple comparison tests using Tukey's adjustment. P-values below 0.05 were considered statistically significant.

\section{Results}

\section{Brain concentrations of quercetin}

Measurements performed one hour after intravenous injection of the nanosomes of quercetin (NSQ) revealed measurable levels of quercetin in the brain (Figure 2). Intraperitoneal injections produced much lower levels.

The peaks for both procedures occurred two hours after the injections, and the peak following intraperitoneal injection was slightly higher. Three hours after quercetin injections, concentrations were low (Figure 2).

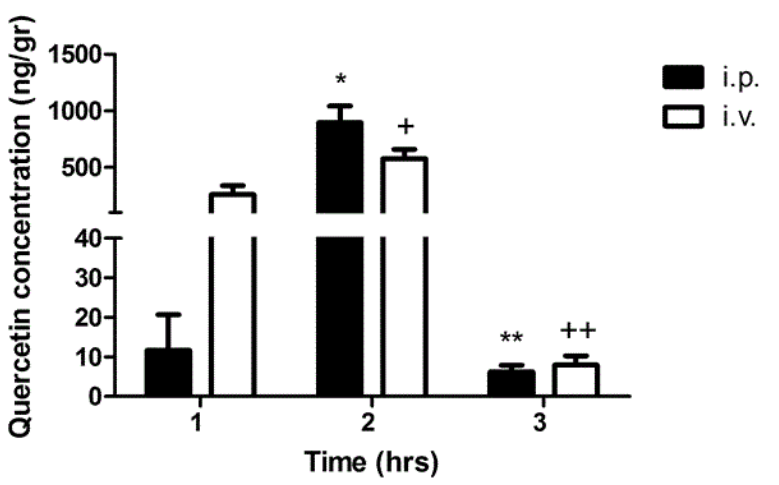

Figure 2: Time course of quercetin brain concentration (ng/g). Rats were injected i.p. or i.v. with $10 \mathrm{mg} / \mathrm{Kg}$ nanosomal preparation of quercetin and 1, 2 or $3 \mathrm{hrs}$ after administration, quercetin levels in the brain were assessed. Control rats without treatment did not show measurable quercetin in brain tissue. Each value is the mean \pm S.D. $\mathrm{P}<0.0001: 1$ hr vs. 2 hrs i.p. $\left(^{*}\right)$ or i.v. $(+), 2$ hrs vs. 3 hrs i.p. $\left(^{* *}\right)$ or i.v. $(++)$.

\section{Lipoperoxidation: Malondialdehyde levels}

The malondialdehyde (MDA) levels in the SN increased significantly $48 \mathrm{hrs}$ after the injection of 6-hydroxydopamine (6OHDA) and decreased following treatments with NSQ administered 1 and $24 \mathrm{hrs}$ after the 6-OHDA treatments. Empty nanosomes (EN) were also effective, and decreased MDA production with a similar time course (Figure 3A).

There was no significant increase in MDA in the corpus striatum (CS), and MDA concentrations were not modified by NSQ or EN administered 1 and 24 hrs after lesioning (Figure 3B).
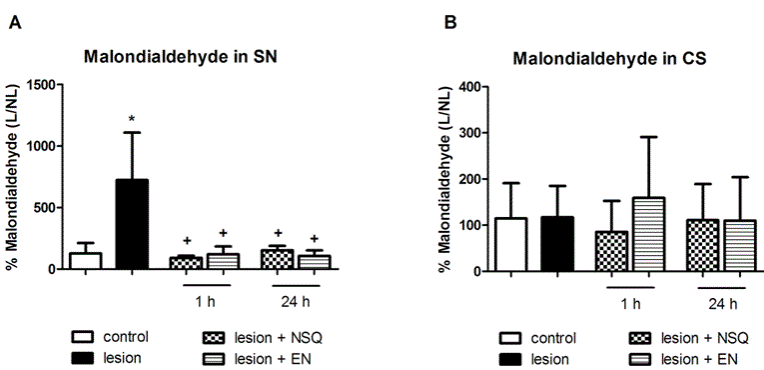

Figure 3: Malondialdehyde levels in SN (A) and CS (B). Rats were injected in the SN with 6-OHDA $(6 \mu \mathrm{g})$. One or $24 \mathrm{hrs}$ after lesion they received $10 \mathrm{mg} / \mathrm{Kg}$ i.p. injection of NSQ or EN. Control rats did not received any treatment. Forty-eight hrs post-lesion malondialdehyde levels were assessed, in the graphs are expressed as the percent (mean + S.D.) of lesion (L)/ no lesion (NL) SN or CS. ${ }^{\star} \mathrm{p}<0.05$ compared to control group. $+\mathrm{p}<0.05$ compared to lesion group. 
Page 5 of 7

\section{Dopamine levels}

In the $\mathrm{SN}$, the $\mathrm{DA}$ levels decreased significantly following the administration of 6-OHDA and did not recover following NSQ or EN treatments that were administered at $1 \mathrm{hr}$ and $24 \mathrm{hrs}$ or following repeated administrations $(1,24,48$ and $72 \mathrm{hrs})$ (Figure $4 \mathrm{~A})$.

In the CS, the DA levels also decreased after lesion and recovered significantly when NSQ was administered $1 \mathrm{hr}$ after 6-OHDA and after repeated doses (1, 24, 48 and $72 \mathrm{hrs})$. EN prevented the DA decrease only following the multiple dose administration scheme (Figure 4B).

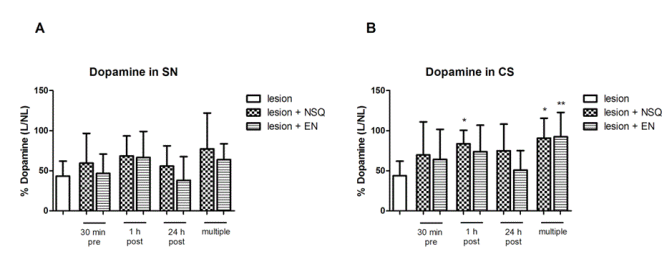

Figure 4: Dopamine levels in SN (A) and CS (B). Rats were injected in the SN with 6-OHDA (6 $\mu \mathrm{g}) .30 \mathrm{~min}$ before, 1,24 or $1,24,48$ and $72 \mathrm{hrs}$ (multiple) after lesion they received $10 \mathrm{mg} / \mathrm{Kg}$ i.p. injection of NSQ or EN. SN and CS dopamine levels were assessed 8 days after lesion and expressed as percent (mean+S.D.) of L/NL side. ${ }^{*} \mathrm{p}<0.05,{ }^{* *} \mathrm{p}<0.01$ compared to lesion group.

\section{Tyrosine hydroxylase-positive neurons}

The number of neurons in the SN that were positive for tyrosine hydroxylase (TH) immunoreactivity was significantly decreased after 6-OHDA administration and did not recover after any of the applied treatments (i.e., NSQ or EN at $1 \mathrm{hr}, 24 \mathrm{hrs}$ or repeated administrations) (Figure $5 \mathrm{~A}$ ). $\mathrm{TH}$ reactivity in the striatum was not altered following any of the treatments (Figure 5B).

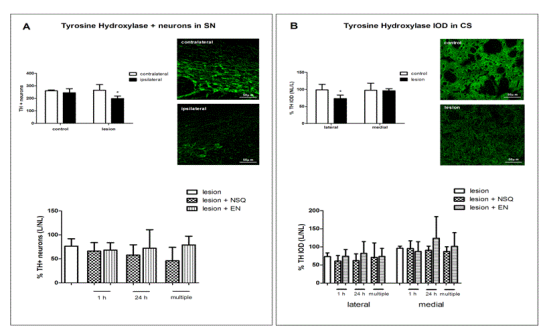

Figure 5: Tyrosine hydroxylase (TH) detection in SN (A) and CS (B). A) Upper graph shows the significant decrease of TH neurons in the ipsilateral related to the contralateral side in lesion animals; at the right there are confocal images of TH neurons. Lower graph shows the percent of $\mathrm{TH}$ neurons in the different treatments. B) Upper graph shows TH IOD in two CS regions, there is a significant decrease of IOD in the lesion compares to control group in the lateral region. No changes were observed in the medial regions; at the right there are confocal images of TH fibers. Lower graph shows TH IOD in the two CS regions after the different treatments. TH positive neurons or TH IOD are expressed as the percent of L/NL side. ${ }^{*} \mathrm{p}<0.05$ ipsilateral vs. contralateral side, control vs. lesion group.

\section{Discussion}

The encapsulation of quercetin in a complex nanosome as utilized in the present study produced measureable brain concentrations of the flavonoid. The intravenous route resulted in the quercetin reaching the brain earlier than did the intraperitoneal route as shown by measurements of the concentrations one hour after the administrations. Both routes of administration (i.e., intravenous and intraperitoneal) resulted in peak concentrations two hours after application.

Studies evaluating the protective effects of quercetin in the brain have focused on antioxidant and protective endpoints and generally have not reported the working concentrations of quercetin. One study estimated a cerebral quercetin concentration of approximate $0.64 \mu \mathrm{M}$ $30 \mathrm{~min}$ after the intraperitoneal administration of liposomal quercetin [8].

Nanosomes are nanometer-sized vesicles of phospholipid bilayers utilized to encapsulate hydrophilic compounds and therapeutic molecules in the aqueous core, whereas hydrophobic compounds and therapeutics such as anticancer and other water insoluble drugs are trapped within the lipid bilayers [19]. Cyclodextrin (CD) as well as cholesterol was added to increase solubility. The most common pharmaceutical application of $\mathrm{CD}$ is to enhance drug solubility in aqueous solutions [20] and $\beta-C D$ can be found in numerous marketed pharmaceuticals. Other quercetin nanosome preparations, with different formulations; e.g., using polyethylene glycol (PEG), have been utilized in testing antitumor activities [21] or to show anxiolytic and cognitive-enhancing effects in rats [14]. Researchers utilizing nanosomes have consistently reported to have better bioavailability and efficacy $[14,21]$.

It is difficult to compare our values with those already published due to the great variability of experimental or testing conditions. Values were obtained with different experimental objectives and doses [14,15] or after chronic administration or acute oral intake (reviewed in 6). With these limitations in mind, our results can be compared with those obtained by Priprem and coworkers for quercetin nanosomes in the rat brain [14].

Forty-eight hours after the administration of 6-OHDA, MDA was significantly increased in the SN but not in the CS. The results in SN confirm the acknowledged oxidative stress provoked by 6-OHDA injection [22]. To understand the contrasting results in the CS, it should be noted that only $50-60 \%$ of the $\mathrm{SN}$ neurons died following 6OHDA administrations in our experimental conditions. The lesions in the CS following such limited injuries are topographically diverse; these lesions are severe in dorsolateral area and minor in the medialcentral regions [23]. Because it is necessary to take all of the CS to achieve detectable levels of MDA for analysis, the less severely lesioned, nearly normal region, likely masks the oxidative process that occurs in the dorsal, most lesioned area. The prevention of the increase in MDA in the SN is a demonstration of the antioxidant capacity of the nanosomal preparation and was an expected result because quercetin is recognized as a potent antioxidant [24].

It was expected that the antioxidant action of the NSQ in the SN 48 hrs after the administration of 6-OHDA would be followed by a protective, survival-enhancing effect; however, as demonstrated by the lack of changes in DA and dopaminergic neuronal survival eight days after the lesion, this was not the case. 
In vitro studies have shown that treatment with quercetin can increase the survival of neurons after lethal oxidative stimuli [8], and neuroprotection due to quercetin treatment has been observed in primary cultures of cortical and hippocampal neurons after a range of cytotoxic insults, including treatments with glutamate, amyloid $\beta$ peptide and $\mathrm{H}_{2} \mathrm{O}_{2}[25,26]$. The administration of quercetin in vivo has not elicited neuroprotection in studies using classical models of experimental PD [11,27] although studies utilizing injections of rotenone in the $\mathrm{SN}$ and quercetin injection over four days have shown antioxidant and protective activities [28].

In agreement with results of the present study and in contrast to the in vitro studies that have produced positive results, quercetin appears to lack strong neuroprotective activity in models of PD in vivo when $\mathrm{SN}$ dopaminergic neuron survival is specifically considered. This conclusion partially contradicts the neuroprotective effects that have been observed following quercetin administration in some models of ischemia and trauma [27-29]. The restricted access of quercetin to the brain, which has been advanced as an explanation, does not apply in our case because; we detected the flavonoid in the brain. Other factors, such as the oxidation of quercetin in the presence of high levels of oxidative stress, should be taken into account; in strongly oxidative conditions, quercetin forms catechol oxidation products, such as semiquinones and quinones, which might alter redox homeostasis and limit the initial positive effects [30]. As 6-OHDA is a highly oxidative molecule and SN lesions have revealed high levels of oxidative stress as observed in the MDA levels, it is likely that the quercetin SN concentrations achieved with the doses utilized were insufficient to reach a maintained antioxidant effect. Indeed, it should be noted that all assessments were performed eight days after the 6-OHDA injections.

The oxidative stress appeared to be lower in the CS than in the SN based on the MDA levels. On the other hand, DA levels in the striatum were decreased following SN lesion but increased significantly following NSQ treatment. These observations can be considered to be indicative of a protective effect, and preliminary results in our laboratory have shown that such observation are accompanied by improvements in the motor activities of the front legs (Prunnel G., personal communication). Lesion-induced reactions of normal and spared terminals that were facilitated by the antioxidative effects of quercetin were presumably responsible for the observed results.

The empty nanosomes also exhibited protective activity following repeated administration. The recognized antioxidant actions of lecithin might explain this activity added to the antioxidant effects of quercetin [31-33]. Studies of markers of oxidative stress other than MDA are currently underway in our laboratory and might shed light on this issue.

Because this study is the first observation of the effects of nanosomes of quercetin, it lacks a dose-response curve that could confirm the hypothesis. The construction of this dose-response curve is currently underway in our laboratory.

\section{References}

1. Nussbaum RL, Ellis CE (2003) Alzheimer's disease and Parkinson's disease. N Engl J Med 348: 1356-1364.

2. Tolleson CM, Fang JY (2013) Advances in the mechanisms of Parkinson's disease. Discov Med 15: 61-66.
3. Hauser DN, Hastings TG (2013) Mitochondrial dysfunction and oxidative stress in Parkinson's disease and monogenic parkinsonism. Neurobiol Dis 51: 35-42.

4. Sian-Hülsmann J, Mandel S, Youdim MB, Riederer P (2011) The relevance of iron in the pathogenesis of Parkinson's disease. J Neurochem 118: 939-957.

5. Martínez-Flórez S, González-Gallego J, Culebras JM, Tunón MJ (2002) [Flavonoids: properties and anti-oxidizing action]. Nutr Hosp 17: 271-278.

6. Joseph JA, Shukitt-Hale B, Denisova NA, Bielinski D, Martin A, et al. (1999) Reversals of age-related declines in neuronal signal transduction, cognitive, and motor behavioral deficits with blueberry, spinach, or strawberry dietary supplementation. J Neurosci 19: 8114-8121.

7. Youdim KA, Joseph JA (2001) A possible emerging role of phytochemicals in improving age-related neurological dysfunctions: a multiplicity of effects. Free Radic Biol Med 30: 583-594.

8. Rivera F, Urbanavicius J, Gervaz E, Morquio A, Dajas F (2004) Some aspects of the in vivo neuroprotective capacity of flavonoids: bioavailability and structure-activity relationship. Neurotox Res 6: 543-553.

9. Dajas F (2012) Life or death: neuroprotective and anticancer effects of quercetin. J Ethnopharmacol 143: 383-396.

10. Datla KP, Christidou M, Widmer WW, Rooprai HK, Dexter DT (2001) Tissue distribution and neuroprotective effects of citrus flavonoid tangeretin in a rat model of Parkinson's disease. Neuroreport 12: 3871-3875.

11. Zbarsky V, Datla KP, Parkar S, Rai DK, Aruoma OI, et al. (2005) Neuroprotective properties of the natural phenolic antioxidants curcumin and naringenin but not quercetin and fisetin in a 6-OHDA model of Parkinson's disease. Free Radic Res 39: 1119-1125.

12. Ossola B, Kääriäinen TM, Männistö PT (2009) The multiple faces of quercetin in neuroprotection. Expert Opin Drug Saf 8: 397-409.

13. Kriinen TM, Piltonen M, Ossola B, Kekki H, Lehtonen S, et al. (2008) Lack of robust protective effect of quercetin in two types of 6hydroxydopamine-induced parkinsonian models in rats and dopaminergic cell cultures. Brain Res 1203: 149-59.

14. Priprem A, Watanatorn J, Sutthiparinyanont S, Phachonpai W, Muchimapura S (2008) Anxiety and cognitive effects of quercetin liposomes in rats. Nanomedicine 4: 70-78.

15. Ghosh A, Mandal AK, Sarkar S, Panda S, Das N (2009) Nanoencapsulation of quercetin enhances its dietary efficacy in combating arsenic-induced oxidative damage in liver and brain of rats. Life Sci 84: 75-80.

16. Zhang Y, Chan HF, Leong KW (2013) Advanced materials and processing for drug delivery: the past and the future. Adv Drug Deliv Rev 65: 104-120.

17. Leonarduzzi G, Testa G, Sottero B, Gamba P, Poli G (2010) Design and development of nanovehicle-based delivery systems for preventive or therapeutic supplementation with flavonoids. Curr Med Chem 17: 74-95.

18. Blasina F, Vaamonde L, Silvera F, Tedesco AC, Dajas F (2015) Intravenous nanosomes of quercetin improve brain function and hemodynamic instability after severe hypoxia in newborn piglets. Neurochem Int 89: 149-156.

19. Blasina F, Vaamonde L, Silvera F, Tedesco AC, Dajas F (2015) Intravenous nanosomes of quercetin improve brain function and hemodynamic instability after severe hypoxia in newborn piglets Neurochem Int. S0197-0186: 30035-30038.

20. Costa G, Abin-Carriquiry JA, Dajas F (2001) Nicotine prevents striatal dopamine loss produced by 6-hydroxydopamine lesion in the substantia nigra. Brain Res 888: 336-342.

21. Paxinos G, Watson G (1986) Rat brain in stereotaxic coordinates Academic Press., Australia.

22. Castor TP (2005) Phospholipid nanosomes. Curr Drug Deliv 2: 329-340. 
Citation: Díaz M, Vaamonde L, Dajas F (2015) Assessment of the Protective Capacity of Nanosomes of Quercetin in an Experimental Model of Parkinson's Disease in the Rat. Gen Med (Los Angel) 3: 207. doi:10.4172/2327-5146.1000207

Page 7 of 7

23. Loftsson T, Brewster ME (1996) Pharmaceutical applications of cyclodextrins. 1. Drug solubilization and stabilization. J Pharm Sci 85: 1017-1025.

24. Yuan ZP, Chen LJ, Fan LY, Tang MH, Yang GL, et al. (2006) Liposomal quercetin efficiently suppresses growth of solid tumors in murine models. Clinical cancer research. Off. J. Am. Assoc. Cancer Res 12: 3193-3199.

25. Glinka Y, Gassen M, Youdim MB (1997) Mechanism of 6hydroxydopamine neurotoxicity. J Neural Transm Suppl 50: 55-66.

26. Urbanavicius J, Ferreira M, Costa G, Abin-Carriquiry JA, Wonnacott S, et al. (2007) Nicotine induces tyrosine hydroxylase plasticity in the neurodegenerating striatum. J Neurochem 102: 723-730.

27. Lakhanpal P, Deepak KR (2007) Quercetin: A versatile flavonoid. Internet Journal of Medical Update 2: 16

28. Arredondo FEC, Blasina F, Vaamonde L, Daz M, Rivera F, et al. (2013) Flavones and flavonols in brain and disease: facts and pitfalls In: Ronald Ross, Watson R (eds) Bioactive nutriceuticals and dietary supplements in neurological and brain disease: prevention and therapy.
29. Dajas F, Rivera-Megret F, Blasina F, Arredondo F, Abin-Carriquiry JA, et al. (2003) Neuroprotection by flavonoids. Braz J Med Biol Res 36: 1613-1620.

30. Karuppagounder SS, Madathil SK, Pandey M, Haobam R, Rajamma U, et al. (2013) Quercetin up-regulates mitochondrial complex-I activity to protect against programmed cell death in rotenone model of Parkinson's disease in rats. Neuroscience 236: 136-148.

31. Schültke E, Kamencic H, Skihar VM, Griebel R, Juurlink B (2010) Quercetin in an animal model of spinal cord compression injury: correlation of treatment duration with recovery of motor function. Spinal Cord 48: 112-117.

32. Boots AW, Haenen GR, den Hartog GJ, Bast A (2002) Oxidative damage shifts from lipid peroxidation to thiol arylation by catechol-containing antioxidants. Biochim Biophys Acta 1583, 279-84.

33. Aabdallah DM, Eid NI (2004) Possible neuroprotective effects of lecithin and alpha-tocopherol alone or in combination against ischemia/ reperfusion insult in rat brain. J Biochem Mol Toxicol 18: 273-278. 